\title{
BEITRÄGE ZUR THEORIE DER SEICHES IN ZUSAMMENGESETZTEN SEEBECKENSYSTEMEN
}

\section{Gerhard Neumann}

In der Natur kommen oft Scen und Meeresbuchten vor, die an einer oder an mehreren Stellen verzweigt sind oder durch enge Kanäle mit anderen Wasserbecken in Zusammenhang stehen. Solche aus einzelnen Teilbecken zusammengesetzten Seen und Meeresbuchten baben, wie die Beobacbtungen an Modellen und natïrlichen Seen gezeigt haben, ilhre ganz besonderen und oft recht komplizierten Schwingungsformen (Seiches). In ihnen bilden sich nicht nur Teilschwingungen der abgeschnürten Seeteile aus, sondern es lassen sich auch Schwingungen des Gesamtsystems nachweisen, die ihrerseits wieder von den Perioden und Dimensionen der abgetrennten Seeteile und der Art und Grosse der Verbindung dieser Teile abhangen.

Der Versuch, die bisher bekannten theoretischen Methoden zur Ermittlung der Eigenperioden auf solche zusanmmengesetzten Seebekkensysteme anzuwenden, führt oft zu Sclıwieringkeiten. Das liegt daran, dass die genannten Theorien von der Voraussetzung ausgehen, dass der Scbwingungsvorgang im ganzen Scliwingungshereich durch ein und dieselbe stetige Funktion der Zeit und des Ortes beschrieben werden kann. Im Falle starker Querschnittsverengungen oder bei engen Ka-

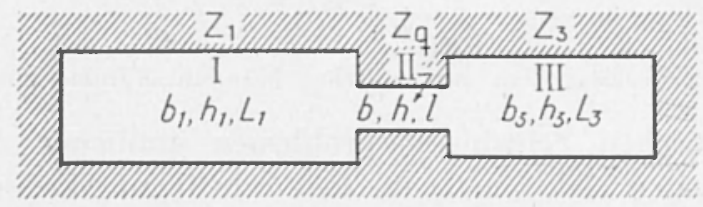

Abb. 1 - Aus zwei Teilbecken zusammengesetztes Seebeckensystem

nalen, die grössere Seebecken miteinander verbinden, ist diese Voraussetzung aber nicht erfüllt. Man wird vielmehr die einzelnen Teile des Seebeckensystems als getrennte Schwingungsgebiete auffassen

(*) La traduzione in lingua italiana della presente nota è riportata a pag. 111 . 
müssen, die sich gegenseitig heeinflussen und durch deren Zusammenwirken (Kopplung) auch freie Schwingungen des Gesamtsystems vorkommen könen. Ist z. B. ein beliebig gestalteter See I an einem Ende durch einen engen Verbindungskanal II mit einem zweiten Wasser. becken III verbunden, wie Abl. 1 schematisch darstellt, dann sind zunachst in jedem Seebecken für sich freie Schwingungen moglich. Nun wird aber bei jedem Anstieg des Seespiegels am unvollständig geschlossenen Ende durch den Verbindungskanal eine gewisse Wassermenge in den benachlbarten Seeteil abstromen und beim Fallen des Seespiegels wieder zurückfliessen. Dadurch wird nicht nur die Eigenperiode der einzelnen Teilbecken in bestimmter Weise verän. dert, sondern es tritt auch eine zusammengesetzte Schwingung des Gesamtsystems auf. Durch die im Verbindungskanal hin und herströmende Wassermenge sind beide Teilbecken miteinander "gekoppelt". Es handelt sich nun darum, neben den Teilschwingungen der einzelnen Seen, auch die Perioden des gekoppelten Gesamtsystems zu bestimmen. Zu diesem Problem gehören eine ganze Reihe anderer, in der Theorie der Seiches wichtiger Falle komplizierter Beckenkombinationen. Eine besondere Rolle spielt die Frage, wie z. B. die Eigenperioden eines Sees verändert werden, wenn der See durch seitliche Offnungen bzw. Kanäle mit dem freien Meer in Verbindung steht. In Abl. 2 ist ein solches seitlich geoffnetes Seebeckensystem schematisch dargestellt, um die Problemstellung zu erläutern.

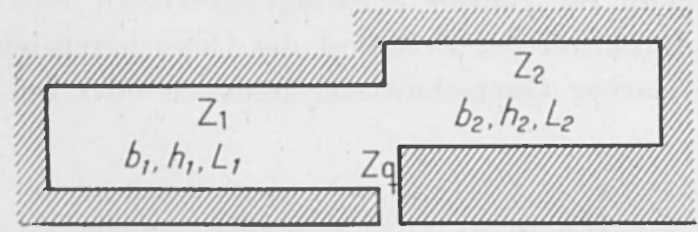

Abb. 2 - Seebeckensystem mit seitlicher Miindungsolfnung zum Meer

Bei akustischen Sclıwingungsproblemen ahnlicher Art und in zusammengesetzten elektromagnetischen Schwingungrkreisen hat man zur Ermittlung der Eigenperioden solcher Systeme mit Erfolg den Begriff der Impedanz eingeführt. Diesen Impedanzbegriff hat Verf ${ }^{(1}$,, vom Beispiel eines scliwingenden Massenpunktes ausgehend, auf kontinuierliche Systeme ïbertragen und sinngemass auf die Theorie der Seiches angewandt. Dahei wurde als hydrodynanische Impedanz bei den Schwingrungen von Wassermassen das Verhältnis

$$
Z=\frac{p_{0}}{S\left(\partial_{\xi} / \partial_{t}\right)_{\text {aax }}}=\frac{\text { Druckamplitude }}{\text { Fläche } \times \text { Geschwindigkeitsamplitude }}
$$


definiert. Diese Grösse ist für einzelne charakteristische Schwingungsgehilde aus den hydrodynamischen Bewegungsgleichungen zu bestimmen. In zusammengesetzten Seebeckensystemen sind die Impedanzen $Z_{1}, Z_{2}, Z_{s}, \ldots$ der Teilglieder des Systems in derselben Weise zu addieren, wie z. B. die Widerstände in elektromagnetischen Sclowingungskreisen. Ist die Impedanz eines Schwingungssystems bekannt, dann lassen sich die Eigenfrequenzen $\omega_{v}=\frac{2 \tau}{T_{v}}$ des Systems aus der Bedingung $Z=O$ bei Vernachlassigung der Reibung gewinnen (sonst $Z=$ Min.).

Für die Impedanz einfacher Schwingungssysteme und ibrer Glieder wurden Formeln abgeleitet $\left({ }^{1}\right)$, die gestatten, die Eigenperiode der Seiches in beliebig gestalteten Seebeckenkombinationen unter Berücksichtigung der unregelmassigen Berkengestalt mit ausreichender Genauigkeit zu berechnen. Sie bilden eine Ergänzung zu den bekannten Seichestheorien, weil sie dort angewandt werden können, wo die letzteren versagen, also bei unvollstandig begrenzten Wassermassen u.s.w.

Für die wichtigsten Glieder eines Schwingungssystems sind die Grössen $Z$ im folgenden angegeben. Bezigglich der Ableitung sei auf die Originalabhandlung $\left.{ }^{(}\right)$verwiesen:

1) Bei einseitig geschlossenen Becken von der mittleren Quersclınittsfläche $S=b h \quad(b--$ Breite, $h=$ Tiefe $)$ gilt

$$
Z=-\frac{i \mathrm{oc}}{s} \operatorname{cotg} \frac{\omega L}{c}, L=\pi / 4
$$

worin $i=\sqrt[3]{-1}, c=\sqrt{g h}, \omega=\frac{2 \pi}{T}, ' L=$ Länge des Beckens, $\lambda=$ Wellenlänge und $\varrho$ die Dichte des $\mathbb{W}^{\prime}$ assers bedeuten. Dieselbe Formel gilt auch für ein beiderseits geschlossenes Becken, nur ist in diesem Falle $i=\hat{n} i_{z}$ zu setzen.

2) Die Impedanz eines an beiden Enden offenen langgestreckten Wasserbeckens ist

$$
Z=\frac{i \varrho c}{S} \operatorname{tg} \frac{\omega L}{c}, L=\lambda / 2
$$

3) Für eine enge Abflussoffnung oder einen engen Kanal vom Querschnitt $q=h b$ und der Länge $L$ gilt

$$
Z_{\mathrm{r}}=\frac{i \mathrm{Q} \omega L}{q} \text {. }
$$


Für die geometrische Lange $L$ des Abflusskanals muss gegebenenfalls eine " effektive" Lünge $L^{\prime}={ }^{\prime} L+\alpha$ eingeführt werlen. Durch die Zusatzstrecke $\iota$ wird die in der Nähe der offnung mitschwingende Wassermasse berïcksichtigt. Es handelt sich hier um die Anbringung einer Art "Mündungskorrektion", ïber die Verf. (") spezielle Untersuchungen angestellt hat und in einer hesonderen Notiz darüher berichten wird.

4. Ein Wasserbecken, das durch eine enge Offnung mit dem freien Meer oder einem anderen grosseren Becken verbunden ist, hat, wenn die Dimensionen dieses Beckens nach allen Richtungen ungefähr gleich sind (kreisförmige Gestalt) eine hesonders bevorzunte Schwingungsform. Es kann zu Schwingungen kommen, bei denen das ganze Wasserniveau im See gleichmassig steigt und fallt. Das akustische Analogon zu diesem Schwingungsgebilde ist der HezmholtzResonator. Die Rechnung ergibt für die Impedanz eines solches Systems

$$
Z=\frac{i_{\varrho} \omega L}{q}-\frac{i_{\varrho} c^{2}}{Q \omega},
$$

worin $Q$ das Volumen des $\mathbb{W}$ asserbeckens bedeutet. $Z$ setzt sich hier aus zwei Teilen zusammen, aus der Impedanz der Abflussöffnung $Z$ (Formel 4) und einem zweiten Gliede $Z=i \varrho c / Q_{(1)}$, der Impedanz des alogeschlossenen $W$ asservolunens. Mit $Z=O$ folgt aus [5]

$$
-\frac{g}{F . \omega}+\frac{\omega L}{q}=0 \quad \text { und } \quad T=2 \pi \sqrt{\frac{F . L}{g \cdot q}}
$$

( $F=$ Areal der Seeoherfläche). Das ist eine in der Theorie der Seiches bekannte und von den Japanern speziell abgeleitete Periodengleicbung, die hier als Nebenergebnis aus der allgemeineren "Impedanztheorie" unmittelhar folgt.

Ganz spezielle Falle hat auch N. ZeiLon $\left(^{(}\right)$behandelt, indem er aus den hydrodynamischen Bewegungsgleichungen unter Berücksich. tigung der Grenzbedingungen die Periode der Seiches in verzweigten Seel)eckensystemen berechnet. Diese älteren Untersuchungen seien hier nur erwähnt, weil sie alle in der vorgeschlagenen Methode unter einheitlichem Gesichtspunkt enthalten sind. Die von ZEILON abgeleiteten Formeln lassen sich mühelos mit Hilfe der Impedanztheorie sofort als Spezialfälle hinschreiben:

a) Verzweigter geschlossener See (vergl. Abb. 3)

An diesem Beispiel lässt sich gleich die Methode erläutern. Der 
See I gabele sich in die Abzweigungen II und III. Bei den Schwingungen kann das $W$ asser aus Becken $I$ sowohl in das Becken II als auch in das Becken III stromen. E's liegen also die Becken II und III mit den Impedanzen $Z_{z}$ und $Z_{3}$ "parallel geschaltet " hinter dem

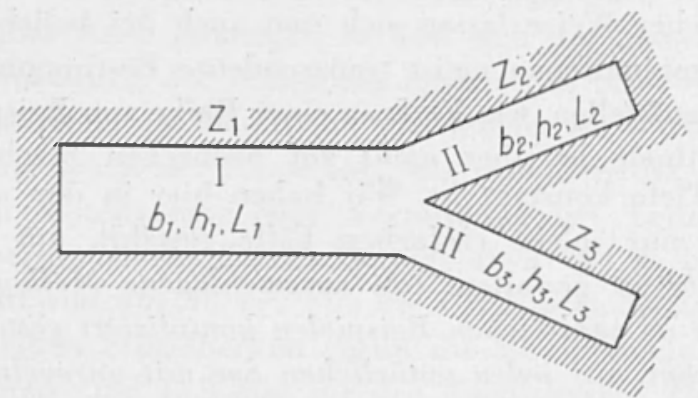

Abb. 3 - Verzweigtes Seebeckensystem

Becken I mit der Impedanz $Z_{1}$. Bezeichnen wir mit $P$ die Gesamtimpedanz von II und III, dann ist

$$
\frac{1}{P}=\frac{1}{Z_{2}}+\frac{1}{Z_{3}} \text { und } P=\frac{Z_{2} Z_{3}}{Z_{2}+Z_{3}}
$$

I und (II + III) sind aher " in Serie geschaltet », d.h.

$$
Z_{1}+P=Z_{1}+\frac{Z_{0} Z_{0}}{Z_{2}+Z_{3}}
$$

Die Bedingung für die Eigenfrequenz wird dann

und mit [2]

$$
\frac{1}{Z_{1}}+\frac{1}{Z_{2}}+\frac{1}{Z_{3}}=0
$$

$$
\frac{b_{1} h_{1}}{\varrho c_{1}}+\frac{\omega L_{1}}{c_{1}}+\frac{b_{9} h_{2}}{\varrho c_{2}}+\frac{\omega L_{2}}{c_{2}}+\frac{b_{3} h_{3}}{\varrho c_{3}}+\frac{\omega L_{3}}{c_{3}}=0 .
$$

Nach Multiplikation mit $g$ (Šchwereheschleunigung) erhalten wir

$$
b_{1} c_{1} \operatorname{tg} \frac{\omega L_{1}}{c_{1}}+b_{2} c_{2} \operatorname{tg} \frac{\omega I_{2}}{c_{2}}+b_{3} c_{3} \operatorname{tg} \frac{\omega I_{3}}{c_{3}}=0,
$$

also die Gleichung von N. ZeiLon ( $\left.{ }^{6}\right)$.

Alınliche transzendente Gleichungen lassen sich auch für den Fall ahleiten, dass einer der Seen, zwei oder alle drei am Ende geoff- 
net sind. Sind z.B. die Seen I und II am Ende geöffnet, See III aher geschlossen, dann folgt

$$
-b_{1} c_{1} \operatorname{cotg} \frac{\omega L_{1}}{c_{1}}-b_{2} c_{2} \operatorname{cotg} \frac{\omega I_{2}}{c_{9}}+\dot{b}_{3} c_{. .} \operatorname{tg} \frac{\omega I_{3}}{c_{3}}=0 .
$$

In ähnlicher Weise lassen sich nun auch bei beliebigen anderen Seebeckenkombinationen meist transzendente Bestimmungsleichungen für $\omega$ bzw. T aufstellen, wie Verf. an einer Reibe von Beispielen gezeigt hat. Die Methode ist aber nicht auf Seebecken beschränkt, deren Breite und Tiefe konstant ist. Wir haben hier in den schematischen Abbildungen nur solche einfachen Falle grewahlt, um das Problem übersichtlicher zu gestalten. Die vorgeschlagene Methode lässt sich, wie Verfasser an zahlreichen Beispiclen konpliziert gestalteter Alpenseen gezeigt hat, auf jeden natürlichen See mit unregelmässiger Konfigaration anvenden. Einige Beispiele mogen zur Erläuterung angefügt sein.

1) Der Königssee in Oberbavern wird durch die Einengung des Beckens bei St. Batholomäi in zwei ungleich grosse Teilbecken zerlegt. Auf ihn lasst sich die schematische Abb. I anwenden. Das gekoppelte Gesamtsystem besteht hier aus zwei Teilseen I und III, die durch einen "Verbindungskanal " II in Zusammenhang stehen. Bezeichnen wir die Impedanzen der Teilglieder der Reihe nach mit $Z_{1}, Z_{q}$ und $Z_{3}(\mathrm{Abl}) .1$, dann ist mit den Formeln [2] und [4] die Bedingung fïr die Eigenfrequenz

und

$$
Z_{1}+Z_{\mathrm{q}}+Z_{3}=-\frac{c_{1}}{S_{1}} \operatorname{cotg} \frac{\omega L_{1}}{c_{1}}+\frac{\omega L}{q}-\frac{c_{3}}{S_{3}} \operatorname{cotg} \frac{\omega I_{i \mathfrak{k}}}{c_{3}}=0
$$

$$
\text { (1) }=\frac{2 \pi}{T}=\frac{q}{L}\left(\frac{c_{1}}{S_{1}} \operatorname{cotg} \frac{\omega L_{i}}{c_{1}}+\frac{c_{*}}{S_{3}} \operatorname{cotg} \frac{\omega L_{3}}{c_{3}}\right) \text {. }
$$

Dafür können wir auch schreiben

$$
\frac{2 \pi}{T}=\frac{q}{L}\left(\frac{c_{1}}{S_{1}} \operatorname{cotg} \pi \frac{T_{1}}{T} \div \frac{c_{\Omega}}{\therefore} \operatorname{cotg} \pi \frac{T_{u 1}}{T}\right) .
$$

In der Gleichung stehen jetzt für $2 L_{1} / c_{1}$ und $2 L_{3} / c_{3}$ die Perioden $T_{1}$ und $T_{11}$ der Teilschwingungen der einzelnen Seen, wie sie bei vollstandig gerlachtem Abschluss an Verbindungskanal auftreten würden. Sie können unter weitgrehender Berücksrichtigungr der Beckenkonfiguration nach der DeFantschen Restmethode ( ${ }^{7}$ ) bestimmt werden. Die in Gleichung [6] auftretende Periode $T$ ist die Eigenperiode des 
gekoppelten Gesamtsystems. Mit $T_{1}$ und $T_{m}$ sind auch die effektiven Werte $c_{1}$ und $c_{3}$ bestimmt, sodass sich $T$ aus der transzendenten Gleichung [6] gerechnen lässt. Für die Hauptperiode des ganzen Königssees ergibt sich $T=11,0 \mathrm{Min}$. Aus Pegelaufzeichnungen hat A. Endrös $\left({ }^{8}\right)$ die Hauptperiode zu 11,6 Min. bestimmt. Die Schwingungen waren aber stark gedämpt, so dass sich die berechnete Periode mit Berücksichtigung der Dämpfung noch etwas erhohen wird.

2) Besonders auffallend sind die Seiches in Waginger-Tachingersee der, wie schon der Name sagt, ein Doppelsee ist. Zwischen beiden Teilseen besteht eine enge Verbindung bei Tettenhausen. Die Lange des verbindenden Kanals beträgt etwa $140 \mathrm{~m}$ bei einem Kanalquerschnitt von nur $90 \mathrm{~m}^{2}$. Für die Grundperiode der Teilschwingungen in beiden Einzelluecken ergibt die Restmethode 18,0 Min für den Wagingersee und 13,8 Min lür den Tachingersee. Die Losung der Gleichung [6] gibt aber für das zusammengesetzte System WagingerTachingerspe eine weitaus grössere Hauptperiode der Seiches namlich $T=64 \mathrm{Min}$. Solche langsamen Schwingungen des Gesamtsystems sind von A. ENDrös $\left({ }^{9}\right)$ neben den kürzeren Teilschwingungen der einzelnen Becken tatsachlich beobachtet worden, obwohl es ihm nicht möglich war, diese auffallend lange Periode theoretisch zu deuten. In den Pegelaufzeichnungen tritt diese Schwingung deutlich mit einer mittleren Periode $T=62 \mathrm{Min}$ in Erscheinung. Diese Seiche erklärt sich also als Hauptschwingung des gekoppelten Systems Waginger-Tachingersee. Das vorliegende Rechenergelonis und seine Ubereinstimmung mit den Beobachtungen erweisen die Brauchbarkeit der hier angewandten Methode. Verf. $\left({ }^{3}\right)$ hat zur Prüfung der Methode noch eine ganze Reihe anderer kompliziert gestalteter Seebeckenkombinationen berangezogen, auf die hier im einzelnen aber nicht eingegangen werden kann. Interessant ist noch das Beispiel des

3) Plan-Heitervangersees. Die Verbindung zwischen dem grosseren Plansee und dem kleineren lleiterwangersee ist extrem eng, wobei der kleine Heiterwangersee mehr als ein "Abflussbecken " für den grösseren Plansee aufzufassen ist. Die Periodengleichung für das zusammengesetzte Seesystem lautet in disem Falle.

$$
\operatorname{cotg} \pi \frac{T_{1}}{T}=\frac{2 \pi S_{1} L}{c_{1} \cdot q} \frac{1}{T}\left(1-\frac{T^{2}}{T_{11}^{2}}\right),
$$

wobei sich die Indizes 1 auf den Plansee beziehen. $T_{1}$ ist die Teilperiode des Plansees und $T_{\mathrm{II}}$ die des Heiterwangersees. $L$ und $q$ sind die Länge und die mittlere Querschnittsflache des verbindenden Ka- 
nals. Die Grundperiode $T$ der Seiches im zusammengesetzten System wird nach Gleichung [7] für die Grundschwingung

$$
T=124 . \text { Minuten, }
$$

also auffallend gross. Auch diese lange Seichesperiode ist von A. ENDRös (10) beobachtet worden, konnte alıer bisher theoretisch scliwer erklärt werden, da die Hauptschwingungsdauer des Plansees allein nur rund $10 \mathrm{Min}$ hetragen würde. FNvRïs vermutete schon eine "Ausgrleichsschwingung " zwischen lıeiden Seen. Dass diese Vermutung richtig ist, bestätigt das vorliegende Ergebnis der Impedanztheorie.

Es ist wohl kaum notig zu bemerken, dass sich aus den Lösungen der transzendenten Periodengleichungen, die für jerle Seeheckenkombination mit Hilfe des Impedanzluegriffes leicht aufgestellt werden können, auch die Perioden der Oberschwingungen lierechnen lassen. Hierauf lıraucht aher in diesem kurzen Referat nicht nälıer eingegangen zu werden.

Eine besondere Rolle kommt den Öfnuıngen an einem Ende oder an der Seite langrgestreckter Seen $z u$, die in ein freies Meeresgebiet iiberleiten (Abh. 2). Durch periodisclıes Abfliessen und Zuströmen von Wasser durch die Offnung werden die Eigenperioden der Seiches im See in hestimmter Weise verindert. Die Impedanztheorie zeigt, dass je nach der Lage der Abflussöffnung zu den Schwingungsbauchen und Knoten der betrachteten Seiche in See eine mehr oder weniger grosse Erniedrigung der Periode eintritt. Am Beispiel des Frischen IIaffes an der ostpreussischen Küste, das durch das enge " Pillauer Tief " mit der Ostsee verlunden ist, konnte gezeigt werden wie stark der Einfluss solcher Offnungen an der Seite schwingender Wassermassen ist. Ohne Berücksichtigung der seitlichen Verlindung zur Ostsee würde die Hauptseiches-Periode des Frischen Ilaffes 9,7 Stunden luetragen. Die vorliegende Theorie ergibt aher bei Mitherücksichtigung des "Pillauer Tiefs" eine Ilauptperiode von nur $T=$ 8,05 Stunden, was mit der heohachteten Seichesperiode von rund 8 Stunden gut ïbereinstimmt.

Diese seitlich oder an Ende geöffneten Seebecken bilden ein Mittelding zwischen "See " und "Meeresbucht ». Sie haben ihre ganz besonderen Seiches. Die mit solchen Becken zusammenliängenden Fragen, insbesondere auch die Frage nach der "Mündungskorrektur » werden in einem speziellen Bericht in dieser Zeitschrift behandelt.

Hamburg - Geophysilalisches Institut der Universität. 


\section{ZUSAMMENF ASSUNG}

Die Anvendung des Impedanzbegriffes auf die Theorie der Seiches führt zu einer einfachen und allgemeinen Methode, die Eigenperioden solcher Seebeckensysteme zu berechnen, die aus mehreren schwingungsfahigen Einzelgebilden zusammengesetzt sind. An Beispielen wird gezeigt, wie die fir bestimmte Beckenkombinationen nach anderen Methoden abgeleiteten I'eriodengleichungen leicht aus der *Impedanztheorie der Seiches * als Spezialfalle herzuleiten sind. Andere, in der Literatur noch nicht behandelte Fälle kompliziert gestalteter Seebeckenkombinationen werden untersucht. Der vorliegende Bericht fasst einige hierher gehörende Arbeiten einheitlich zusammen und zeigt, wie z. B. die Eigenperiode eines Sees, der durch enge Kanäle mit anderen IV asserbecken verbundeu ist, durch die Abzweigungen beeinflusst wird. Die Methode ist nicht auf Seebecken konstanter Breite und Tiefe beschränkt, sondern lässt sich auf jeden unregelmässig gestalteten See anwenden, wie an Beispielen der Alpenseen gezeigt wird.

\section{BIBLIOGRAFIA}

(1) Necmann G.: Uber die Periode freier Schwingungen in zwei durch eincn engen Kanal miteinander verbundenen Seen. Ann. Hydr. u. mar. Metor., 1943, Seite 409 .

(-) - Die Impedanz mechanischer Schtoingungssysteme und ihre Anwendung auf die Theorie der Seiches. Ann. Hydr. u. mar. Meteor., 1944, S. 65.

(3) - Eine Methode zur Berechnung der Eigenperioden zusammengesetzter (gekoppelter) Seebeckensysteme. Ann. Hydr. u. mar. Meteor., 1944, S. 193.

(4) - Freie Schwingungen (Seiches) der Putziger Wiek. Ann, Hydr. u. mar. Meteor., 1944, S. 225.

(5) - Uber Resonanzschwingungen von Meeresbuchten und die Mundungskorrektur bei Seiches. Deutsche Hydr. Zeitschr., Bd. 1, Heft 2/3, 1948.

(9) ZeILon N.: On the seiches of the Gullmar Fjord. Ur Svenska Hydr.-Biol. Komm. Skrifter, V, 1913.

(7) Defant A.: Neue Methode zur Ermittlung der Eigenschwingungen (Seiches) von abgeschlossenen Wassernassen. Ann. Hydr. u. mar. Meteor., 1918, S. 78.

(8) Endros A.: Eine merkwürdige Seiche des Königssees und die eigentumliche Temperaturschichtung seines Tiefenwassers. Pet. Geogr. Mitt., 1927, S. 73.

(9) - Die Seiches des Waginger-Tachingersees. Sitz. ber. Math.Phys. KI. d. Bayr. Akademie d. Wiss, Bd. 35, Heft 3, 1905.

(10) - Beobachtungen über die Dampfung der Seiches in Seen. Gerl. Beitr. z. Geophysik, Bd. 41, 1934. 